\title{
Exotic Higgs Decays (Theory)
}

\section{Elina Fuchs $\mathbf{s}^{a, b, c, *}$}

${ }^{a} C E R N$,

Department of Theoretical Physics, 1211 Geneve 23, Switzerland

${ }^{b}$ Leibniz Universität Hannover,

QuantumFrontiers, Institute for Theoretical Physics, Appelstraße 2, 30167 Hannover, Germany

${ }^{c}$ Physikalisch-Technische Bundesanstalt,

QuantumFrontiers, Bundesallee 100, 38116 Braunschweig, Germany

E-mail: elina.fuchs@cern.ch

A plethora of New Physics models introduces new decay modes of the discovered Higgs boson. This talk presents a short overview of different models with exotic Higgs decays as well as current bounds and sensitivities at future colliders, taking direct searches and indirect constraints from global Higgs coupling fits into account.

The Ninth Annual Conference on Large Hadron Collider Physics - LHCP2021

7-12 June 2021

Online

${ }^{*}$ Speaker 


\section{Introduction}

With just $5 \%$ of its planned data, the LHC has already performed precision studies of the production and decay modes of the discovered Higgs boson with a mass of $m_{h}=125 \mathrm{GeV}$. Of particular interest is the question whether it has additional decay channels beyond those in the Standard Model (SM). These could significantly alter its total width. The reasons for the small Higgs width of $\Gamma_{h}^{\mathrm{SM}}=4.1 \mathrm{MeV} \approx O\left(10^{-5}\right) m_{h}$ are: the partial widths into SM particles are $(i)$ loop-suppressed such as $h \rightarrow \gamma \gamma, Z \gamma, g g$, (ii) phase space-suppressed in the case of the decay into a pair of massive vector bosons, $H \rightarrow W W^{*}, Z Z^{*}$ or even kinematically forbidden in the case of the top quark as the SM particle with the largest interaction with the Higgs, or (iii) coupling-suppressed for the decay into a pair of fermions where even the largest branching ratio, $\mathrm{BR}^{\mathrm{SM}}(h \rightarrow b \bar{b}) \approx 58 \%$ stems from a small Yukawa coupling of $y_{b}^{\mathrm{SM}} \approx 2 \cdot 10^{-2}$. The Higgs width determined via the on-shell to off-shell cross section ratio of $H \rightarrow Z Z^{*} \rightarrow 4 \ell$ [1] yields $\Gamma_{h}=3.2_{-2.2}^{+2.8} \mathrm{MeV}$ [2], in agreement with the SM, but with large uncertainties while a precision of $\sim 20 \%$ is expected at the HL-LHC [3]. Various opportunities are pursued and planned at the LHC and future colliders to look directly or indirectly for Higgs decays into new particles [4].

\section{Models with exotic Higgs decays}

In many models, the Higgs boson acts as a window to New Physics and is therefore expected to decay into new particles $X$ if $2 m_{X}<m_{h}$, for example in the SM augmented by an additional scalar singlet $(\mathrm{SM}+\mathrm{S})$, fermion(s) $(\mathrm{SM}+\psi)$, the $2 \mathrm{HDM} / \mathrm{MSSM}+\mathrm{S}$, as well as Little Higgs and Hidden Valleys [4]. In particular, the Higgs boson can be a portal to a Dark Sector [5-7] via the interaction of the Higgs with the new scalar $\Phi$. The most general scalar potential of the $\mathrm{SM}+\mathrm{S}$, $V_{\mathrm{s}}(\Phi, H)=V(\Phi)+\mu^{2}(\Phi) H^{\dagger} H+\lambda_{h}\left(H^{\dagger} H\right)^{2}$, with $H=(0, v+h)^{T} / \sqrt{2}$ and $\Phi=\phi_{0}+\phi$ (choosing $\phi_{0}=0$ ), is realized as in Tab. 1 by the renormalizable real singlet extension (without a $\mathbb{Z}_{2}$ symmetry of $\Phi)$ [8-11] where $\mathrm{s}_{\theta} \equiv \sin \theta$ is the $\phi-h$ mixing angle and $c_{h \phi \phi}$ is the dimensionful trilinear coupling of $h \phi \phi$. The relaxion [12], slowly rolling down a linear potential $\left(r>1 /(4 \pi)^{2}, g \ll 1\right.$, $f \geq \Lambda \geq 1 \mathrm{TeV}$ ) before the periodic backreaction term $\tilde{M}$ stops it at $\phi_{0}$ corresponding to the observed Higgs mass, is a special case of the $\mathrm{SM}+\mathrm{S}$. The $h-\phi$ mixing $[13,14]$ can naturally be larger for the relaxion [15] than for the real singlet. The relaxion collider phenomenology is determined by $m_{\phi}$ and $\mathrm{s}_{\theta}[11,16]$ whereas $\lambda_{h \phi}$ and $a_{h \phi}$ depend on them. The tree-level width of the Higgs into NP is given by $\Gamma_{h \rightarrow \phi \phi}=\frac{c_{h \phi \phi}^{2}}{8 \pi m_{h}} \sqrt{1-\frac{4 m_{\phi}^{2}}{m_{h}^{2}}}$, see Fig. 1 .

\begin{tabular}{c|c|c}
\hline \hline & renormalizable singlet & relaxion \\
\hline$V(\Phi)$ & $-a_{h \phi} v^{2} \Phi+\frac{1}{2} m_{0}^{2} \Phi^{2}+\frac{a_{\phi}}{3} \Phi^{3}+\frac{\lambda_{\phi}}{4} \Phi^{4}$ & $r g \Lambda^{3} \Phi$ \\
$\mu^{2}(\Phi)$ & $-\mu_{0}^{2}+2 a_{h \phi} \Phi+\hat{\lambda}_{h \phi} \Phi^{2}$ & $-\Lambda^{2}+g \Lambda \Phi-\tilde{M}^{2} \cos \left(\frac{\Phi}{f}\right)$ \\
$\mathrm{s}_{\theta}$ & $\approx \frac{a_{h \phi}}{v \lambda_{h}}$ & $\approx \frac{\tilde{M}^{2}}{2 v f \lambda_{h}} \sin \left(\frac{\phi_{0}}{f}\right)$ \\
$c_{h \phi \phi}$ & $\approx \frac{1}{2} \mathrm{~s}_{\theta}^{2} \frac{m_{h}^{2}}{v}+\lambda_{h \phi} v$ & $\approx \frac{3}{2} \mathrm{~s}_{\theta} \frac{m_{h}^{2}}{v}+\frac{m_{\phi}^{2}}{v}$ \\
\hline \hline
\end{tabular}

Table 1: Comparison of the renormalizable real singlet extension and the relaxion case, approximations holds for $m_{\phi}^{2} \ll m_{h}^{2}$, 

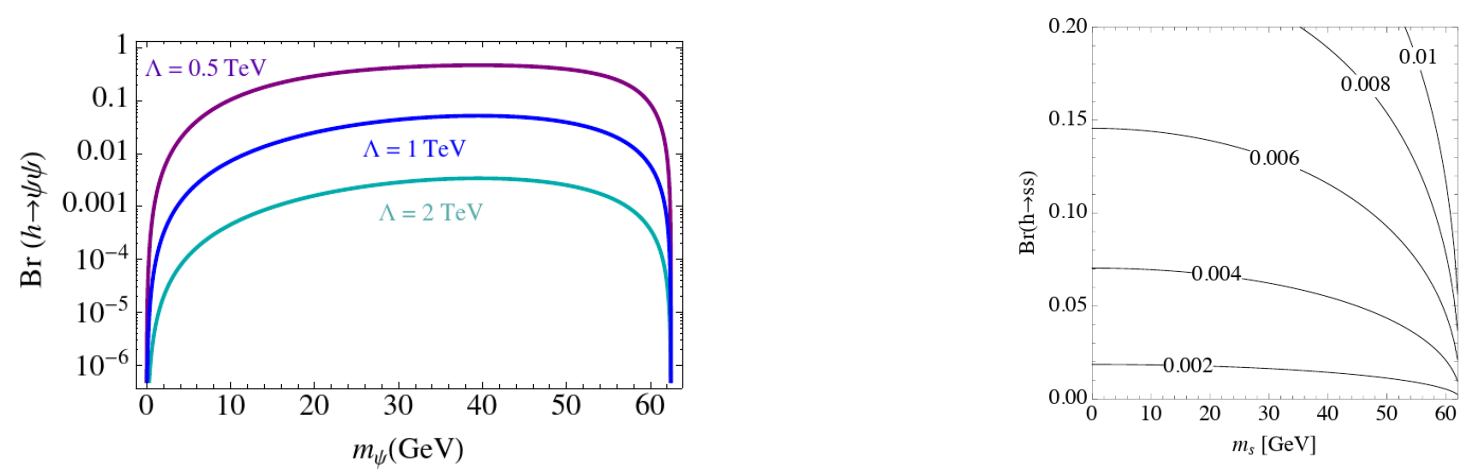

Figure 1: Predicted BR $\mathrm{BSM}_{\mathrm{B}}$ : (left) $\mathrm{SM}+\psi$ with $\Delta \mathcal{L}=\frac{\mu}{\Lambda^{2}}|H|^{2} \bar{\psi} \psi$ for $\mu=m_{\psi}$ different values of the NP scale $\Lambda ;$ (right) $\mathrm{SM}+\mathrm{S}(s \equiv \phi)$ for different values of $c_{h \phi \phi}[4]$.

A flavor off-diagonal Higgs decay, $h \rightarrow f_{1} \bar{f}_{2}$, would be a clear sign of NP. Models such as 2HDMs, extra dimensions and compositeness and flavor frameworks like Minimal Flavor Violation or a Frogatt-Nielsen symmetry can accommodate flavor changing Higgs decays [17-20]. Recently, CMS stringently constrains lepton-flavor violating Higgs decays to $\mathrm{BR}(h \rightarrow \mu \tau, e \tau)<$ $0.15 \%, 0.22 \%$, respectively [21], and ATLAS constrains $\operatorname{BR}(h \rightarrow e \mu)<0.0061 \%$ [22].

\section{Indirect bounds on an exotic decay width}

Searching for signatures of an exotic decay $h \rightarrow X X$ with missing transverse energy $E_{T}$ constrains the invisible width. Moreover, untagged decay products, such as light jets, are not necessarily undetectable, but not accounted for among the tagged visible Higgs decay channels. The BSM width is the sum, $\Gamma_{\mathrm{BSM}} \equiv \Gamma(h \rightarrow \mathrm{BSM})=\Gamma_{\text {inv }}+\Gamma_{\text {unt }}$. The searches for $h \rightarrow E_{T}+X$ yield $\mathrm{BR}_{\text {inv }}<11 \%$ at $95 \% \mathrm{CL}$ [23]. Under the mild assumption on $\Gamma_{h}^{\text {tot }}$ of $\kappa_{W}, \kappa_{Z} \leq 1$ in the $\kappa$-framework [24], which holds e.g. in any doublet or singlet extension, this bound can be improved in a global fit by a combination with the precise measurements of the signal strengths of the visible production and decay channels, resulting in $\mathrm{BR}_{\mathrm{inv}}<9 \%$ [25]. The HL-LHC can reach $\mathrm{BR}_{\text {inv (unt) }}<1.9(4.0) \%$, and combined with the FCC-ee/eh/hh even $\mathrm{BR}_{\text {inv (unt) }}<0.024(1.0) \%$ [26]. $\mathrm{BR}_{\text {inv }}$ can be applied on models with Higgs decay products that are stable on the detector scale.

\section{Direct searches for exotic decay products}

Fig. 2 shows direct searches for the decays of $X$ (here as light pseudoscalars $h \rightarrow a a$ ) into 4 lighter SM particles. ATLAS [27] and CMS [28] cover the mass range from $1 \mathrm{GeV}$ to $m_{h} / 2$ with several channels. The delayed arrival of a jets originating from long-lived $X \mathrm{~s}$ was proposed in Ref. [29] to enhance the sensitivity to $\operatorname{BR}(h \rightarrow X X)$ depending on the lifetime $\tau_{X}$ by means of a timing layer with nanosecond precision, see Fig. 3. Furthermore, Ref. [30] highlights Higgs decays into displaced jets in the clean environment of the $\mathrm{LHeC}$.

For the $\mathrm{SM}+\mathrm{S}$ and relaxion, Fig. 4 compares the $\mathrm{BR}_{\mathrm{BSM}}, \mathrm{BR}_{\text {unt }}$ and (strong if applicable) $\mathrm{BR}_{\mathrm{inv}}$ bounds. In the long-lived region, the displaced vertex (DV) searches and the timing of delayed jets with the MTD timing layer [29] are very sensitive. The purple dotted lines of $\lambda_{h \phi}$ and $s_{\theta}^{2}$ indicates the natural parameter space below. The relaxion can only be realized on the blue thick line. 
Tagging exotic Higgs decays into many $b$-jets with deep neural networks has been proposed in Ref. [31] to bridge the general bound on $h \rightarrow \mathrm{BSM}$ and stronger exclusive direct searches.
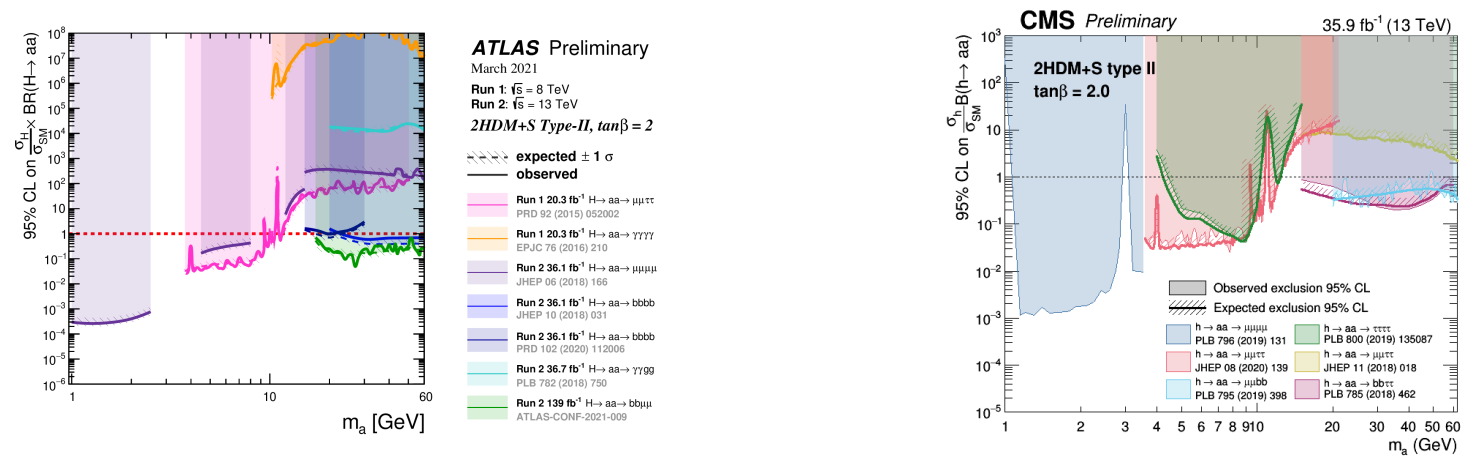

Figure 2: Summary of the direct searches for exotic Higgs decays into light pseudoscalars $a$ incl. Run-2 data at ATLAS [27] (left) and CMS [28] (right) in various final states.
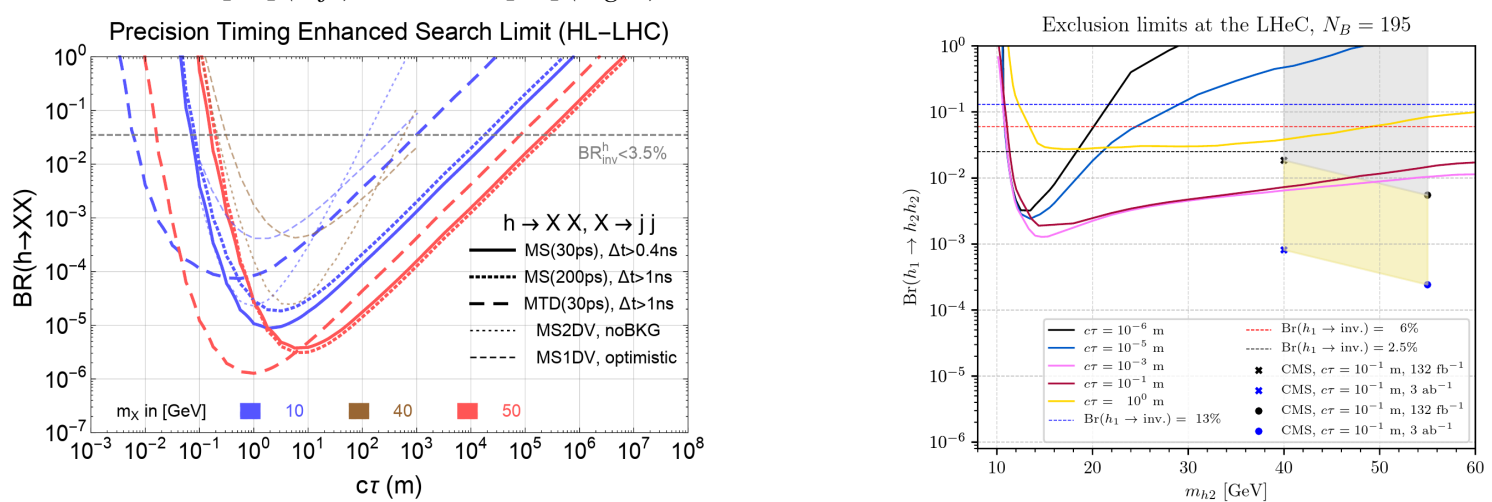

Figure 3: Proposals to detect long-lived Higgs decay products: (left:) Delayed jets with the muon spectrometer (MS) and MIP (Minimally Ionizing Particle) Timing Detector (MTD) at the HL-LHC [29]. (right:) Sensitivity to decays into lighter Higgs bosons $h_{2}$ at the LHeC assuming 195 background events [30].

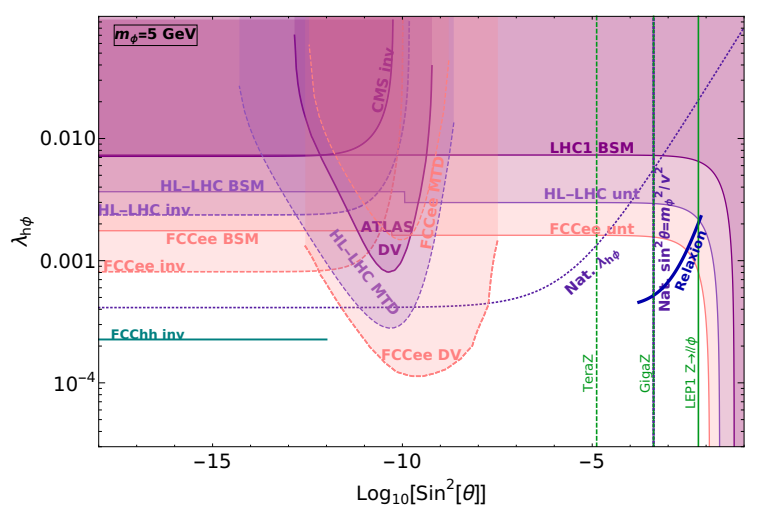

Figure 4: Constraints (solid) and projections (dashed) on the exotic decay $h \rightarrow \phi \phi$ in the SM+real singlet $(\phi)$ extension with $\lambda_{h \phi} \equiv \hat{\lambda}_{h \phi}+\mathrm{s}_{\theta} \frac{a_{\phi}}{v}$ vs the relaxion (thick blue line) from Ref. [11] with a mass of $m_{\phi}=5 \mathrm{GeV}$, comparing bounds on Higgs decays into any BSM, untagged or invisible decay products ( $h \rightarrow \mathrm{BSM}$, unt, inv) with displaced vertices (DV), the MTD timing layer, and $Z$-factory measurements (green), at the (HL-)LHC and FCC-ee/hh.

Summary Exotic decays of the discovered Higgs boson arise in a plethora of generic portals and well-motivated models and are a thus vibrant opportunity to search for BSM effects - directly via prompt, displaced/delayed or invisible signatures, or indirectly via global fits. 
I thank the LHCP2021 conveners and organizers, my collaborators of $[11,13,16]$, and the Minerva and Humboldt Foundations, Fermilab, the University of Chicago and the Weizmann Institute for the support during those projects.

\section{References}

[1] F. Caola and K. Melnikov, "Constraining the Higgs boson width with ZZ production at the LHC,” Phys. Rev. D 88 (2013), 054024 [arXiv:1307.4935 [hep-ph]].

[2] A. M. Sirunyan et al. [CMS], "Measurements of the Higgs boson width and anomalous $H V V$ couplings from on-shell and off-shell production in the four-lepton final state," Phys. Rev. D 99 (2019) no.11, 112003 [arXiv:1901.00174 [hep-ex]].

[3] M. Cepeda, S. Gori, P. Ilten, M. Kado, F. Riva, R. Abdul Khalek, A. Aboubrahim, J. Alimena, S. Alioli and A. Alves, et al. "Report from Working Group 2: Higgs Physics at the HL-LHC and HE-LHC," CERN Yellow Rep. Monogr. 7 (2019), 221-584 [arXiv:1902.00134 [hep-ph]].

[4] D. Curtin, R. Essig, S. Gori, P. Jaiswal, A. Katz, T. Liu, Z. Liu, D. McKeen, J. Shelton and M. Strassler, et al. "Exotic decays of the $125 \mathrm{GeV}$ Higgs boson," Phys. Rev. D 90 (2014) no.7, 075004 [arXiv:1312.4992 [hep-ph]].

[5] B. Patt and F. Wilczek, "Higgs-field portal into hidden sectors," [arXiv:hep-ph/0605188 [hep$\mathrm{ph}]]$.

[6] O. Lebedev, “The Higgs portal to cosmology," Prog. Part. Nucl. Phys. 120 (2021), 103881 [arXiv:2104.03342 [hep-ph]].

[7] S. Argyropoulos, O. Brandt and U. Haisch, "Collider Searches for Dark Matter through the Higgs Lens," [arXiv:2109.13597 [hep-ph]].

[8] F. Piazza and M. Pospelov, "Sub-eV scalar dark matter through the super-renormalizable Higgs portal,” Phys. Rev. D 82 (2010), 043533 [arXiv:1003.2313 [hep-ph]].

[9] P. Huang, A. J. Long and L. T. Wang, "Probing the Electroweak Phase Transition with Higgs Factories and Gravitational Waves," Phys. Rev. D 94 (2016) no.7, 075008 [arXiv:1608.06619 [hep-ph]].

[10] B. Heinemann and Y. Nir, "The Higgs program and open questions in particle physics and cosmology,” Usp. Fiz. Nauk 189 (2019) no.9, 985-996 [arXiv:1905.00382 [hep-ph]].

[11] E. Fuchs, O. Matsedonskyi, I. Savoray and M. Schlaffer, "Collider searches for scalar singlets across lifetimes," JHEP 04 (2021), 019 [arXiv:2008.12773 [hep-ph]].

[12] P. W. Graham, D. E. Kaplan and S. Rajendran, "Cosmological Relaxation of the Electroweak Scale," Phys. Rev. Lett. 115 (2015) no.22, 221801 [arXiv:1504.07551 [hep-ph]].

[13] T. Flacke, C. Frugiuele, E. Fuchs, R. S. Gupta and G. Perez, "Phenomenology of relaxionHiggs mixing," JHEP 06 (2017), 050 [arXiv:1610.02025 [hep-ph]]. 
[14] K. Choi and S. H. Im, "Constraints on Relaxion Windows," JHEP 12 (2016), 093 [arXiv: 1610.00680 [hep-ph]].

[15] A. Banerjee, H. Kim, O. Matsedonskyi, G. Perez and M. S. Safronova, "Probing the Relaxed Relaxion at the Luminosity and Precision Frontiers,” JHEP 07 (2020), 153 [arXiv:2004.02899 [hep-ph]].

[16] C. Frugiuele, E. Fuchs, G. Perez and M. Schlaffer, "Relaxion and light (pseudo)scalars at the HL-LHC and lepton colliders," JHEP 10 (2018), 151 [arXiv:1807.10842 [hep-ph]].

[17] J. L. Diaz-Cruz and J. J. Toscano, "Lepton flavor violating decays of Higgs bosons beyond the standard model," Phys. Rev. D 62 (2000), 116005 doi:10.1103/PhysRevD.62.116005 [arXiv:hep-ph/9910233 [hep-ph]].

[18] R. Harnik, J. Kopp and J. Zupan, "Flavor Violating Higgs Decays," JHEP 03 (2013), 026 [arXiv:1209.1397 [hep-ph]].

[19] J. Kopp and M. Nardecchia, "Flavor and CP violation in Higgs decays," JHEP 10 (2014), 156 [arXiv:1406.5303 [hep-ph]].

[20] A. Dery, A. Efrati, Y. Nir, Y. Soreq and V. Susič, "Model building for flavor changing Higgs couplings," Phys. Rev. D 90 (2014), 115022 doi:10.1103/PhysRevD.90.115022 [arXiv:1408.1371 [hep-ph]].

[21] L. Morvaj [ATLAS and CMS], "Higgs rare and exotic decays," [arXiv:2105.06951 [hep-ex]].

[22] G. Aad et al. [ATLAS], "Search for the Higgs boson decays $H \rightarrow e e$ and $H \rightarrow e \mu$ in $p p$ collisions at $\sqrt{s}=13 \mathrm{TeV}$ with the ATLAS detector," Phys. Lett. B 801 (2020), 135148 [arXiv:1909.10235 [hep-ex]].

[23] [ATLAS], "Combination of searches for invisible Higgs boson decays with the ATLAS experiment," ATLAS-CONF-2020-052.

[24] S. Heinemeyer et al. [LHC Higgs Cross Section Working Group], "Handbook of LHC Higgs Cross Sections: 3. Higgs Properties,” [arXiv:1307.1347 [hep-ph]].

[25] [ATLAS], "A combination of measurements of Higgs boson production and decay using up to $139 \mathrm{fb}^{-1}$ of proton-proton collision data at $\sqrt{s}=13 \mathrm{TeV}$ collected with the ATLAS experiment," ATLAS-CONF-2020-027.

[26] J. de Blas, M. Cepeda, J. D’Hondt, R. K. Ellis, C. Grojean, B. Heinemann, F. Maltoni, A. Nisati, E. Petit and R. Rattazzi, et al. "Higgs Boson Studies at Future Particle Colliders," JHEP 01 (2020), 139 [arXiv:1905.03764 [hep-ph]].

[27] [ATLAS], "Summary of Exotic Higgs Boson Decays from the ATLAS Experiment," ATLPHYS-PUB-2021-008.

[28] [CMS], "Summary of 2HDM+S searches at $13 \mathrm{TeV}$ (Run 2 - 2016)". 
[29] J. Liu, Z. Liu and L. T. Wang, "Enhancing Long-Lived Particles Searches at the LHC with Precision Timing Information,” Phys. Rev. Lett. 122 (2019) no.13, 131801 [arXiv:1805.05957 [hep-ph]].

[30] K. Cheung, O. Fischer, Z. S. Wang and J. Zurita, "Exotic Higgs decays into displaced jets at the LHeC," JHEP 02 (2021), 161 [arXiv:2008.09614 [hep-ph]].

[31] S. Jung, Z. Liu, L. T. Wang and K. P. Xie, "Probing Higgs exotic decay at the LHC with machine learning," [arXiv:2109.03294 [hep-ph]]. 